PHYSICAL REVIEW D 95, 109905(E) (2017)

\title{
Erratum: Photon-photon dispersion of TeV gamma rays and its role for photon-ALP conversion \\ [Phys. Rev. D 91, 083003 (2015)]
}

Alexandra Dobrynina, Alexander Kartavtsev, and Georg Raffelt

(Received 9 May 2017; published 30 May 2017)

DOI: $10.1103 /$ PhysRevD.95.109905

To obtain the spectral average of the refraction effect in Eq. (11), we have erroneously averaged over the number distribution rather than the energy distribution of the background photons, i.e., we have used $x^{2}$ in the integrand of Eq. (11) instead of $x^{3}$ and the concomitant normalization factor $1 /\left(2 \zeta_{3}\right)$ instead of $15 / \pi^{4}$. The correct equation is

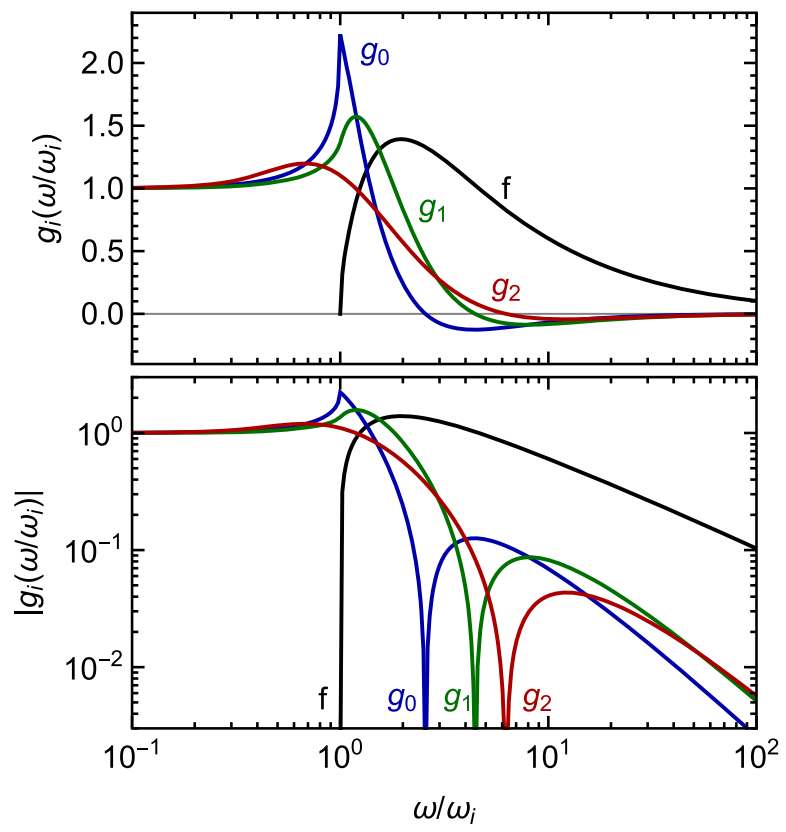

FIG. 1. The red line has changed as a result of the corrected Eq. (11).

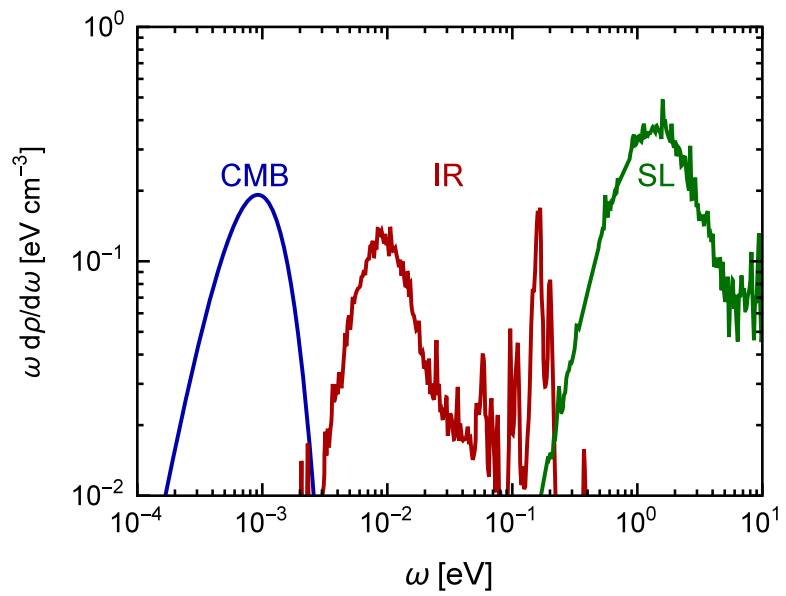

FIG. 2. The shape of the cosmic microwave background (CMB) curve has changed, reflecting the modified Eq. (11), whereas the other curves have remained unchanged. 


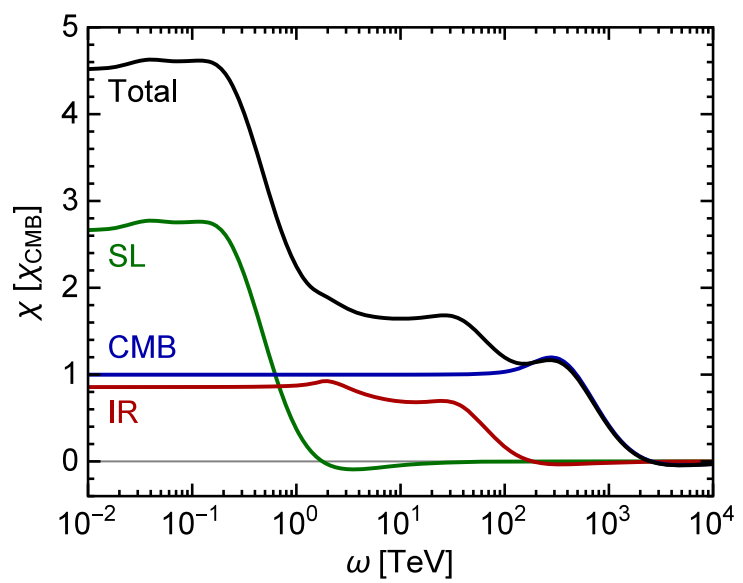

FIG. 3. The shape of the shoulder of the CMB curve has slightly changed as well as the corresponding region of the "total" curve.

$$
g_{2}(w)=\frac{15}{\pi^{4}} \int_{0}^{\infty} d x \frac{x^{3}}{e^{x}-1} g_{1}\left(w x \frac{30 \zeta_{3}}{\pi^{4}}\right)
$$

We thank Hendrik Vogel and Ranjan Laha for spotting this error. This correction slightly changes the figures, but the overall conclusions of the paper remain the same. 